\title{
Growth of Acidithiobacillus ferrooxidans ATCC 23270 in thiosulfate under oxygen-limiting conditions generates extracellular sulfur globules by means of a secreted tetrathionate hydrolase
}

\author{
Simón Beard ${ }^{1+}$, Alberto Paradela ${ }^{2}$, Juan P.Albar ${ }^{2}$ and Carlos A. Jerez ${ }^{1 *}$ \\ ' Faculty of Sciences, Laboratory of Molecular Microbiology and Biotechnology, Department of Biology, Millenium Institute for Cell Dynamics and Biotechnology, \\ University of Chile, Santiago, Chile \\ 2 Servicio de Proteómica, Centro Nacional de Biotecnología, Consejo Superior de Investigaciones Científicas, Madrid, España
}

\section{Edited by:}

Thomas E. Hanson, University of

Delaware, USA

\section{Reviewed by:}

Biswarup Mukhpadhyay, Virginia Bioinformatics Institute, USA

John Stolz, Duquesne University, USA

${ }^{*}$ Correspondence:

Carlos A. Jerez, Facultad de Ciencias,

Departamento de Biología, Universidad

de Chile, Santiago 1, Casilla 653,

Santiago, Chile.

e-mail: cjerez@uchile.cl

t'Present address:

Simón Beard, Biotecnologías

Antofagasta S.A., Las Araucarias

9080-9110 módulo C, Quilicura,

Santiago, Chile.

e-mail: sbeard@bta-sa.cl
Production of sulfur globules during sulfide or thiosulfate oxidation is a characteristic feature of some sulfur bacteria. Although their generation has been reported in Acidithiobacillus ferrooxidans, its mechanism of formation and deposition, as well as the physiological significance of these globules during sulfur compounds oxidation, are currently unknown. Under oxygen-sufficient conditions (OSC), A. ferrooxidans oxidizes thiosulfate to tetrathionate, which accumulates in the culture medium. Tetrathionate is then oxidized by a tetrathionate hydrolase $(T \mathrm{TH})$ generating thiosulfate, elemental sulfur, and sulfate as final products. We report here a massive production of extracellular conspicuous sulfur globules in thiosulfate-grown A. ferrooxidans cultures shifted to oxygen-limiting conditions (OLC). Concomitantly with sulfur globule deposition, the extracellular concentration of tetrathionate greatly diminished and sulfite accumulated in the culture supernatant. A. ferrooxidans cellularTTH activity was negligible in OLC-incubated cells, indicating that this enzymatic activity was not responsible for tetrathionate disappearance. On the other hand, supernatants from both OSC- and OLC-incubated cells showed extracellular TTH activity, which most likely accounted for tetrathionate consumption in the culture medium. The extracellular TTH activity described here: (i) gives experimental support to the TTH-driven model for hydrophilic sulfur globule generation, (ii) explains the extracellular location of $A$. ferrooxidans sulfur deposits, and (iii) strongly suggests that the generation of sulfur globules in A. ferrooxidans corresponds to an early step during its adaptation to an anaerobic lifestyle.

Keywords: Acidithiobacillus ferrooxidans, sulfur globules, extracellular tetrathionate hydrolase, thiosulfate oxidation

\section{INTRODUCTION}

During oxidation of reduced inorganic sulfur compounds (RISCs), by some microaerophilic chemotrophic sulfur bacteria and by anoxygenic phototrophic microorganisms, the deposition of intracellular or extracellular conspicuous sulfur globules as an important (and sometimes obligatory) intermediary occurs. It has been proposed that production of obligated intracellular sulfur deposits, during anoxygenic sulfide and thiosulfate oxidation in photolithotrophic and facultative anaerobic chemolithotrophic bacteria, correlates with the lack of sulfur dehydrogenase (SoxCD), normally present in the thiosulfate-oxidizing multi-enzyme system (TOMES; Friedrich et al., 2005). The latter is a characteristic feature of microorganisms that oxidize RISCs by using the branched Paracoccus sulfur oxidation (PSO) pathway (Grimm et al., 2008; Ghosh and Dam, 2009, and references therein). The mechanisms for production of sulfur globules by sulfur bacteria that do not use the branched-PSO pathway are currently unknown. Acidithiobacillus ferrooxidans is a facultative anaerobe, chemolithoautotroph gram negative $\gamma$-proteobacterium that obtains its energy from the oxidation of ferrous iron, elemental sulfur, or RISCs, amongst other compounds (Pronk et al., 1990; Rohwerder and Sand, 2003; Rawlings, 2005). Due to its ability to solubilize metal sulfides, this bacterium has been successfully applied in biomining operations (Olson et al., 2003; Rawlings, 2005). In A. ferrooxidans, thiosulfate is oxidized through the S4-intermediary pathway (S4I; Pronk et al., 1990; Kelly et al., 1997; Ghosh and Dam, 2009). The first step in the S4I-pathway is the condensative oxidation of two molecules of thiosulfate to generate the four sulfur atoms intermediate tetrathionate, a reaction catalyzed by a thiosulfate dehydrogenase (TD). The second step in the S4I-pathway is tetrathionate hydrolysis by a tetrathionate hydrolase (TTH), generating sulfate and disulfane monosulfonic acid $\left(\mathrm{HS}_{2} \mathrm{SO}_{3}^{-}\right)$as direct hydrolysis products. Due to the high reactivity of the last compound, A. ferrooxidans TTH generates sulfate, thiosulfate, and elemental sulfur as final products (de Jong et al., 1997). This enzyme has been purified and its corresponding coding gene (tetH; BAF03501) was identified (Buonfiglio et al., 1999; Kanao et al., 2007). A cyclic thiosulfate-oxidizing pathway has been proposed in thiobacilli, in which disulfane monosulfonic acid is biologically oxidized to trithionate $\left(\mathrm{S}_{3} \mathrm{O}_{6}^{2-}\right)$, and then hydrolyzed to thiosulfate and sulfate by means of a trithionate hydrolase without the production of long-chain polythionates or elemental sulfur (Pronk et al., 1990; Ghosh and Dam, 2009). However, the production of long-chain polythionates and elemental sulfur has been reported 
in aerated A. ferrooxidans suspensions incubated with tetrathionate (Steudel et al., 1987). Based on these results, a set of reactions involving the highly reactive disulfane monosulfonic acid were proposed, in which this compound reacts with itself in successive elongation chain reactions leading to the production elemental sulfur and sulfite $\left(2 \mathrm{HS}_{2} \mathrm{SO}_{3}^{-} \leftrightarrow \mathrm{HS}_{4} \mathrm{SO}_{3}^{-}+\mathrm{HSO}_{3}^{-} ; 2 \mathrm{HS}_{4} \mathrm{SO}_{3}^{-} \leftrightarrow \mathrm{HS}_{8} \mathrm{SO}_{3}^{-}\right.$ $+\mathrm{HSO}_{3}^{-} ; \mathrm{HS}_{8} \mathrm{SO}_{3}^{-} \leftrightarrow \mathrm{S}_{8}^{\circ}+\mathrm{HSO}_{3}^{-}$; Figure 1).

The hydrophobic elemental sulfur generated can be "solubilized" by polythionates in a micelle-like structure, forming hydrophilic sulfur globules (Steudel et al., 1987). Due to the reversibility of the reactions involved in the higher polythionates pathway and because their formation does not explain oxidation of the sulfanesulfur moiety from thiosulfate, the physiological significance of polythionates (and hence of hydrophilic sulfur globules) as intermediates during sulfur oxidation remains obscure (Kelly et al., 1997; Ghosh and Dam, 2009). The appearance of visible sulfur (sulfur globules) during $A$. ferrooxidans growth in a substratelimited chemostat on tetrathionate under high substrate concentrations (above $400 \mu \mathrm{M}$ ) has been reported. Transmission electron microscopy (TEM) micrographs of these cells showed tiny globules located between the inner and outer membranes or in a discrete cytoplasmic vacuole-like structure (Hazeu et al., 1988). Despite the low concentration and small size of sulfur globules produced by thiosulfate-grown A. ferrooxidans, it was possible to identify polythionates as the main sulfur species present in them by using sulfur K-edge X-ray absorption near-edge spectroscopy (XANES) analysis (Prange et al., 2002). However, some controversy exist regarding the methodological approach used by Prange et al. (2002) in their sulfur K-edge XANES analysis (George et al., 2008) and other researchers have found elemental sulfur as the main sulfur species in A. ferrooxidans sulfur globules (He et al., 2010).

Sulfur globule deposition is a characteristic feature amongst anoxygenic sulfur bacteria. In the proposed model for hydrophilic sulfur globule production, TTH is the key enzyme involved and it does not use molecular oxygen during tetrathionate hydrolysis. The reaction proceeds under both aerobic and anaerobic conditions (de Jong et al., 1997). Moreover, disulfane monosulfonic acid can lead to the formation of polythionates both in aerobic and anaerobic conditions $\left(2 \mathrm{HS}_{2} \mathrm{SO}_{3}^{-}+1 / 2 \mathrm{O}_{2} \rightarrow \mathrm{S}_{6} \mathrm{O}_{6}^{2-}+\mathrm{H}_{2} \mathrm{O} ; 2 \mathrm{HS}_{2} \mathrm{SO}_{3}^{-} \leftrightarrow \mathrm{S}_{5} \mathrm{O}_{6}^{2-}+\mathrm{H}_{2} \mathrm{~S}\right.$; Figure 1; Steudel et al., 1987), indicating that hydrophilic sulfur globule forming reactions may proceed under anoxygenic conditions. Therefore, the effect of oxygen availability during thiosulfate oxidation by $A$. ferrooxidans was studied in the present communication. Thiosulfate-grown A. ferrooxidans cultures shifted to oxygen-limiting conditions (OLC) produced extracellular sulfur globules. In addition, extracellular TTH activity was detected in the growth medium of OLC-incubated A. ferrooxidans cells and the corresponding protein was identified by mass spectrometry analysis. Extracellular sulfur globules formed under OLC contained elemental sulfur and concomitantly with their production sulfite was also accumulated in the growth medium. Therefore, the extracellular TTH activity and the chemical by-products detected here give experimental support to the TTH-driven hydrophilic sulfur globule production model proposed by Steudel et al. (1987) and suggest for the first time a mechanism for extracellular sulfur globule deposition by an S4I-pathway-using microorganism. Furthermore, our results suggest that deposition of sulfur globules might be an early step of a physiological response to a decrease in oxygen availability.

\section{MATERIALS AND METHODS \\ BACTERIAL STRAINS AND GROWTH CONDITIONS}

Acidithiobacillus ferrooxidans strain ATCC 23270 was grown in batch cultures by using thiosulfate-containing DSMZ medium 71 [in grams per liter: $\mathrm{KH}_{2} \mathrm{PO}_{4}, 3.0 ; \mathrm{MgSO}_{4} \cdot 7 \mathrm{H}_{2} \mathrm{O}, 0.5 ;\left(\mathrm{NH}_{4}\right)_{2} \mathrm{SO}_{4}$, 3.0; $\left.\mathrm{CaCl}_{2} \cdot 2 \mathrm{H}_{2} \mathrm{O}, 0.25 ; \mathrm{Na}_{2} \mathrm{~S}_{2} \mathrm{O}_{3} \cdot 5 \mathrm{H}_{2} \mathrm{O}, 5.0 ; \mathrm{pH} 4.4-4.7\right]$. No ferrous sulfate was added to the medium according to the instructions given by DSMZ. A. ferrooxidans cells were washed with basal salts DSMZ 71 medium solution and inoculated at a density of $2.5 \times 10^{6}$ cells/mL in Erlenmeyer flasks ( $2 \mathrm{~L}$ ) containing $500 \mathrm{~mL}$ of DSMZ 71 medium (flask to culture volume ratio of 0.25 ) followed by incubation at $30^{\circ} \mathrm{C}$ with shaking (150 RPM). Once the culture reached the mid to late-exponential-phase, shifts to OLC were achieved by

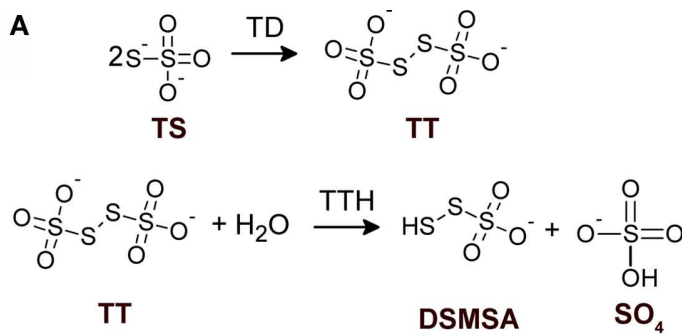

B

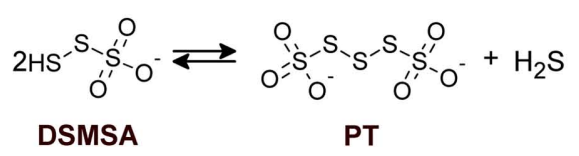

FIGURE 1 | Detailed structures of relevant sulfur compounds and reactions proposed for sulfur globules production. (A) Enzymatically catalyzed reactions. TD, thiosulfate dehydrogenase; TTH, tetrathionate hydrolase. (B) Anoxygenic condensation of disulfane monosulfonic acid that leads to polythionates generation. (C) Successive condensation reactions of

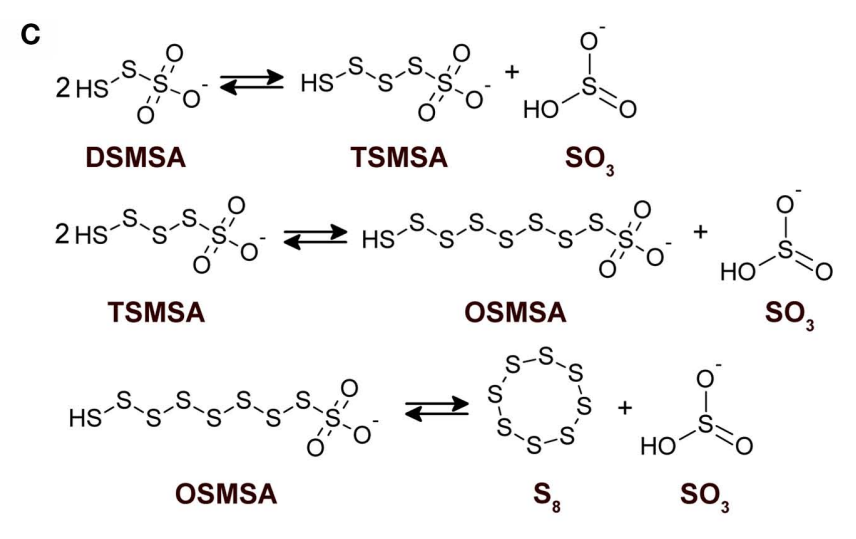

sulfane monosulfonic acids that leads to elemental sulfur production. TS, thiosulfate; TT, tetrathionate; DSMSA, disulfane monosulfonic acid; $\mathrm{SO}_{4^{\prime}}$ sulfate; $\mathrm{SO}_{3}$, sulfite; $\mathrm{PT}$, pentathionate; TSMSA, tetrasulfane monosulfonic acid; OSMSA, octosulfane monosulfonic acid; $\mathrm{S}_{8^{\prime}}$ elemental sulfur. Reactions according to Steudel et al. (1987). 
trespassing 200-250 $\mathrm{mL}$ of the grown culture to a $250-\mathrm{mL}$ flask (culture to flask volume ratio of $0.8: 1.0$ ) followed by incubation at $30^{\circ} \mathrm{C}$ without shaking. The remaining A. ferrooxidans culture was kept in the 2-L Erlenmeyer flask (culture to flask volume ratio of 0.1:0.125) and was further incubated at $30^{\circ} \mathrm{C}$ with shaking. These cultures were used as the oxygen-sufficient condition (OSC). Cell and sulfur globule counting was carried out by using a PetroffHausser chamber in a phase-contrast Olympus BX50 microscope. Sulfur globules were identified as extracellular refringent structures viewed at a $600 \times$ magnification. After centrifugation of OLC- and OSC-cultures $\left(5,000 \times g\right.$ at $4^{\circ} \mathrm{C}$ for $\left.20 \mathrm{~min}\right)$ media supernatants were filtered through a $0.22-\mu \mathrm{m}$ nitrocellulose filter and were used to determine presence of different thiols and enzymatic activities.

\section{PREPARATION OF CELL-FREE EXTRACTS}

Acidithiobacillus ferrooxidans cells were washed three times by centrifugation with $1 \mathrm{M}$ ammonium sulfate $\mathrm{pH} 3.0$ and finally resuspended in $500 \mu \mathrm{L}$ of $1 \mathrm{M}$ ammonium sulfate $\mathrm{pH}$ 3.0. To obtain cell-free extracts, cells were disrupted by sonication on ice (Misonix XL2020, Farmingdale, NY, USA; 20\% intensity cycles of 20 s on, $40 \mathrm{~s}$ off for $10 \mathrm{~min}$ total time). Unbroken cells and cellular debris were removed by centrifugation at $15,000 \times g$ at $4^{\circ} \mathrm{C}$ for $10 \mathrm{~min}$.

\section{ANALYSIS OF SULFUR COMPOUNDS}

Thiosulfate was determined by the decoloration of methyleneblue at $670 \mathrm{~nm}$ in acidic conditions (Kletzin, 1989). Tetrathionate was determined by a cold-cyanolysis protocol (Kelly et al., 1969). Production of extracellular sulfur in A. ferrooxidans cultures was estimated by the increase in absorbance at $430 \mathrm{~nm}$ (Hazeu et al., 1988). Elemental sulfur was quantitatively determined by extraction with acetone/water (19:1) followed by cyanolysis (Kelly and Wood, 1998) and normalized by the amount of total protein content of the cell pellet extracted. Analysis of low molecular thiols present in A. ferrooxidans growth medium supernatants was carried out by using HPLC after sample derivatization with THIOLYTE monobromobimane reagent (Calbiochem, La Jolla, CA, USA) as previously described (Rethmeier et al., 1997).

\section{OXYGEN DETERMINATION}

Oxygen measurements were carried out in a liquid-phase oxygraph (Hansatech Instruments, Ltd., Norfolk, UK). The electrode cell was filled with $2.5 \mathrm{~mL}$ of a mid to late-exponential-phase culture of A. ferrooxidans and the oxygen concentration in the medium was recorded. To mimic oxygen-suficient conditions, the electrode chamber lid was removed and $1.0 \mathrm{~mL}$ of the A. ferrooxidans culture was kept in the cell chamber. At different times, $0.1 \mathrm{~mL}$ aliquots were taken and the absorbance at $430 \mathrm{~nm}$ was measured.

\section{ENZYMATIC ASSAYS}

Thiosulfate dehydrogenase activity was determined spectrophotometrically by using ferricyanide as an artificial electron acceptor. The reaction was followed by the thiosulfate-dependent decrease in ferricyanide absorbance at $420 \mathrm{~nm}$. The TD reaction mixture contained (in $1 \mathrm{~mL}$ final volume): $150 \mathrm{mM}$ potassium acetate buffer $\mathrm{pH}$ 5.0, $1 \mathrm{mM}$ ferricyanide, $10 \mathrm{mM}$ sodium thiosulfate, and $50 \mu \mathrm{g} \mathrm{mL} \mathrm{m}^{-1}$ of cell-free extract protein. One unit of activity $(\mathrm{U})$ was defined as the amount of enzyme that catalyzes the reduction of $1 \mu \mathrm{mol}$ ferricyanide $\mathrm{min}^{-1}$.
Tetrathionate hydrolase activity was determined in a continuous assay, measuring the increase in absorbance at $290 \mathrm{~nm}$ due to the production of long-chain sulfur intermediates derived from tetrathionate hydrolysis (de Jong et al., 1997). The reaction mixture contained (in $0.1 \mathrm{~mL}$ final volume): $1 \mathrm{M}$ ammonium sulfate $\mathrm{pH} 3.0,1 \mathrm{mM}$ sodium tetrathionate, and $50 \mu \mathrm{g} \mathrm{mL}^{-1}$ of cell-free extract protein. TTH activity was determined in aliquots of filtrated growth medium supernatants from $A$. ferrooxidans cultures incubated under OLC and OSC. Units for TTH activity were expressed as $\Delta \mathrm{Abs} 290 \mathrm{~nm} \mathrm{~min}^{-1}$. All enzymatic reactions were carried out at room-temperature. Data presented for sulfur compounds and enzymatic activities correspond to the mean values and SD of at least three independent experiments.

\section{RNA MANIPULATIONS}

Acidithiobacillus ferrooxidans total RNA was prepared as previously reported for qRT-PCR analysis (Navarro et al., 2009). Primers tetHFw (TGCTGTTCCAATGGATTCAA) and tetHR1 (TGATACGCCGTTCCTTATCC) were used for tet $H$ amplification and 16SFw (TGGTGCCTAGCGTACTGAGTG) and 16SR (CCGAAGGGCACTTCCGCA) for A. ferrooxidans $16 \mathrm{~S}$ rRNA. Absolute copy numbers for tet $H$ and $16 \mathrm{~S}$ rRNA were determined by using standard curves obtained by serial dilutions of $A$. ferrooxidans genomic DNA. Copy numbers of tet $H$ relative to $16 \mathrm{~S}$ rRNA were determined at each OLC-incubation time from three independent experiments and the mean values and SD are shown.

\section{PRECIPITATION OF EXTRACELLULAR PROTEINS AND THEIR IDENTIFICATION BY MASS SPECTROMETRY ANALYSIS}

Acidithiobacillus ferrooxidans cells were separated from their growth medium $(400 \mathrm{~mL})$ by centrifugation at $5,000 \times g$ at $4^{\circ} \mathrm{C}$ for $20 \mathrm{~min}$. Proteins present in the filtered supernatants were precipitated by using the PRMM method (Caldwell and Lattemann, 2004) as applied to A. ferrooxidans cells (Pagliai and Jerez, 2009). Precipitated proteins were electrophoresed on a Tris/Glycine SDS polyacrylamide gel and stained with Coomassie Blue G-250. Bands of interest were excised manually from the gels and the proteins present in them were identified by MALDI peptide mass fingerprinting and MALDI-TOF/TOF MS analysis as described before (Varela et al., 2010). Possible subcellular localization of identified proteins was predicted by using the SubCell 1.0 server at Center for Biological Sequence Analysis, BioCentrum-DTU, The Technical University of Denmark (http://www.cbs.dtu.dk/services/SubCell/). Protein measurements were carried out by using Bradford's method (PIERCE, Rockford, IL, USA) and crystalline BSA as standard.

\section{RESULTS GROWTH OF A. FERROOXIDANS IN THIOSULFATE AS ENERGY SOURCE}

As it has been previously shown (Hazeu et al., 1986) during thiosulfate oxidation by $A$. ferrooxidans, tetrathionate was accumulated in the culture medium, and in our experimental conditions it reached a maximum concentration of c.a. $5 \mathrm{mM}$ (Figure 2A). The ratio between the rates of thiosulfate consumption and tetrathionate increase in the culture medium was c.a. 4:1. This ratio, and the stoichiometric relationship involved in tetrathionate formation by means of thiosulfate oxidation by TD, suggests that half of the tetrathionate formed from thiosulfate oxidation diffuses out of the cells and into the culture medium. Tetrathionate concentration 

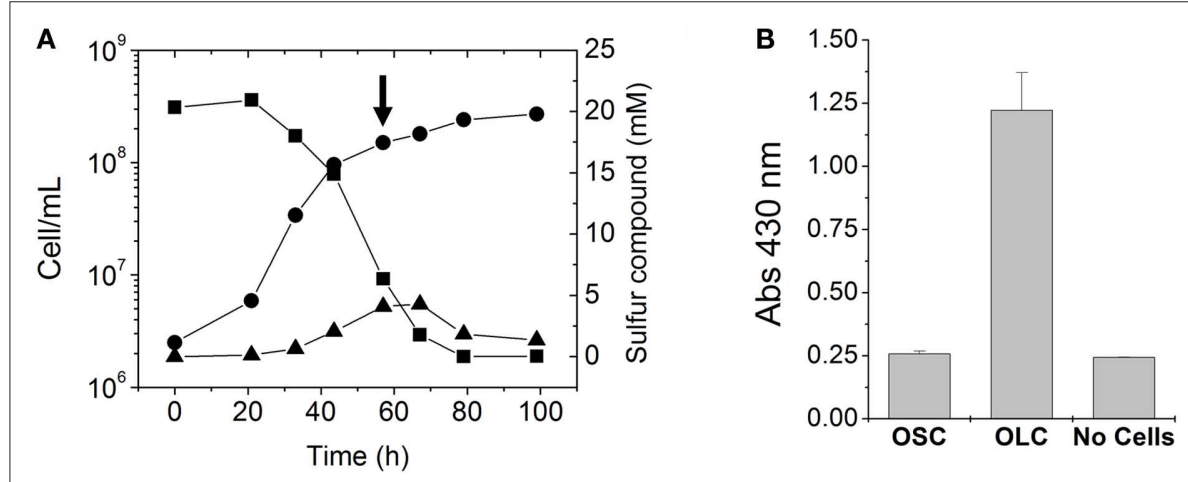

C

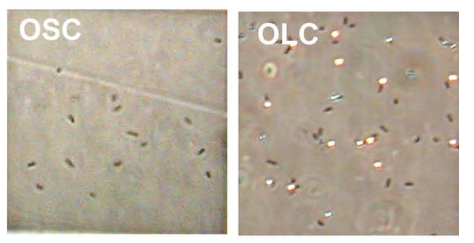

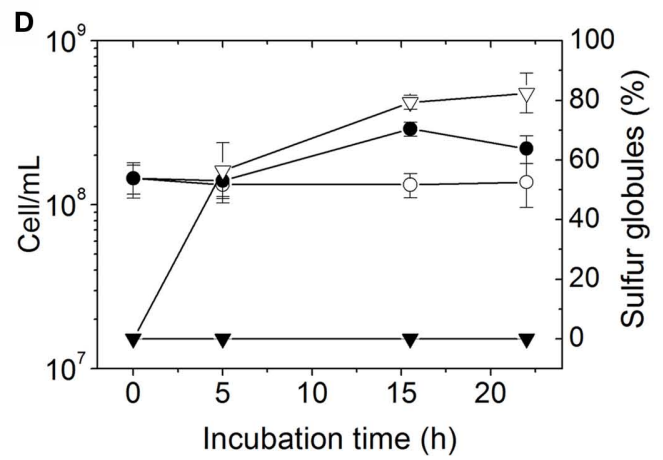

FIGURE 2 | Production of extracellular sulfur globules by $A$. ferrooxidans during incubation under oxygen-limiting conditions. (A) A. ferrooxidans was grown in thiosulfate under OSC and the changes in concentrations of thiosulfate $(\boldsymbol{\square})$ and tetrathionate $(\mathbf{\Lambda})$ during $A$. ferrooxidans cell growth $(\bullet)$ were determined. To study the effect of oxygen limitation, thiosulfate-grown $A$. ferrooxidans cultures were either shifted to OLC or kept under OSC at the point of the growth curve shown by an arrow. (B) Generation of elemental sulfur as detected by the increase in the absorbance at $430 \mathrm{~nm}$ in A. ferrooxidans cultures. Three $A$. ferrooxidans cultures were grown in thiosulfate until the late-exponential-phase. One culture was subjected for $16 \mathrm{~h}$ to OSC and another one to OLC. The third culture was filtered $(0.22 \mu \mathrm{m}$-filter) and the medium obtained was incubated for $16 \mathrm{~h}$ under OLC and was used as the "No Cells" control. (C) Phase-contrast micrographies (600 $\times$ magnification) of $A$. ferrooxidans cells from OLC- and OSC-incubated cultures shown in (B). (D) Cell growth of $A$. ferrooxidans cultures $(\bullet, O)$ and percentages of sulfur globules produced $(\boldsymbol{\nabla}, \nabla)$ during OSC- $(\bullet, \nabla)$ or OLC- $(O, \nabla)$ incubations. began to decrease only when thiosulfate was almost completely consumed (Figure 2A). Most likely, tetrathionate starts being oxidized by cells due to the lack of thiosulfate as an energy source.

\section{EFFECT OF OXYGEN AVAILABILITY IN THIOSULFATE-GROWN A. FERROOXIDANS CULTURES}

When mid to late-exponential-phase thiosulfate-grown A. ferrooxidans cultures were shifted to OLC, their media became white and their absorbance at $430 \mathrm{~nm}$ was significantly increased compared to OSC-incubated cultures (Figure 2B). The increment in absorbance at $430 \mathrm{~nm}$ suggests the production of elemental sulfur in OLCincubated cultures. This change in absorbance was not observed when cells were removed by filtration before shifting the culture to OLC (Figure 2B), indicating that the increase in absorbance was not due to the chemical reactivity of the thiosulfate/tetrathionate mixture present in the culture medium when the OLC-incubation started, but rather to a cell-dependent phenomenon. Moreover, after $20 \mathrm{~h}$ incubation of $A$. ferrooxidans under OSC or OLC $\mathrm{pH}$ values of culture supernatants were $2.30 \pm 0.51$ and $3.23 \pm 0.44$, respectively, indicating that the increment in absorbance at $430 \mathrm{~nm}$ observed was not due to a low $\mathrm{pH}$-dependent decomposition of thiosulfate. Inspection of cells under the phase-contrast microscope showed a massive production of extracellular globular-shaped refringent structures (sulfur globules) only in OLC-incubated cultures (Figure 2C). Cell growth and production of sulfur globules were both directly counted under the phase-contrast microscope at different times during OSC- or OLC-incubations. 50\% of the total specimens (bacteria and sulfur globules) counted by phase-contrast microscopy corresponded to sulfur globules after incubating cells for $5 \mathrm{~h}$ under OLC. They reached a maximum of $80 \%$ after incubating for $15 \mathrm{~h}$. On the other hand, production of sulfur globules was not observed at all in cultures incubated under OSC (Figure 2D).

\section{EXTRACELLULAR A. FERROOXIDANS SULFUR GLOBULES CONTAIN ELEMENTAL SULFUR}

The increase in absorbance at $430 \mathrm{~nm}$ observed in A. ferrooxidans cultures shifted to OLC (Figure 2B) suggests the production of elemental sulfur in the medium. To determine whether the extracellular globules produced by $A$. ferrooxidans contained elemental sulfur, A. ferrooxidans cells pelleted together with globules were extracted with acetone and sulfur was determined by cyanolysis. During incubation under OLC, the amount of sulfur extracted increased, whereas under OSC sulfur amounts remained unchanged (Figure 3A). Moreover, visual inspection of acetone-extracted $A$. ferrooxidans cells by phase-contrast microscopy showed that sulfur globules were completely extracted from OLC-incubated cultures 
after acetone treatment (Figure 3B). These results confirm that the refringent structures produced during OLC-incubations corresponded to elemental sulfur-containing globules.

\section{SULFUR GLOBULE PRODUCTION DEPENDS ON OXYGEN CONCENTRATION}

To correlate extracellular sulfur globule production with the availability of oxygen, the concentration of oxygen dissolved in a thiosulfate-grown culture of $A$. ferrooxidans was determined by using a liquid-phase oxygraph. The absorbance at $430 \mathrm{~nm}$ of the culture incubated in the oxygraph-cell was recorded and used as an indicator of the production of sulfur globules. Oxygen initially dissolved in the culture medium dropped within the first $15 \mathrm{~min}$ of incubation, most likely due to its consumption by A. ferrooxidans cells. After the medium was depleted of oxygen absorbance at $430 \mathrm{~nm}$ begun to increase (Figure 4). Moreover, when oxygen concentration increased again (by opening the oxygraph-cell lid), a concomitant decrease in absorbance at $430 \mathrm{~nm}$ was obtained (Figure 4). This suggests that sulfur compounds accumulated during OLC-incubation were readily oxidized most likely by $A$. ferrooxidans cells once oxygen was restored.

\section{ANALYSIS OF SULFUR COMPOUNDS OXIDATION DURING SULFUR GLOBULES PRODUCTION}

To determine the mechanisms underlying extracellular sulfur globule production under OLC-incubations, concentrations of thiosulfate and tetrathionate in culture media, and enzymatic activities related to its oxidation (TD and TTH) under OLC- and OSC-incubations were measured. Thiosulfate was completely depleted from the culture medium after $25 \mathrm{~h}$ OSC-incubation (Figure 5A), almost certainly due to its consumption by the cells. Under OLC-incubation, thiosulfate concentration slightly increased within the first $5 \mathrm{~h}$, and after that period it remained unchanged (Figure 5A). TD activity of cells incubated under OLC was c.a. six times lower than that of cells incubated under OSC $(0.15 \pm 0.06$ and $0.99 \pm 0.08 \mathrm{U} \mathrm{mg}$ protein $^{-1}$, respectively; Figure 5C). This result was expected since thiosulfate oxidation was not observed in the OLC-incubated culture

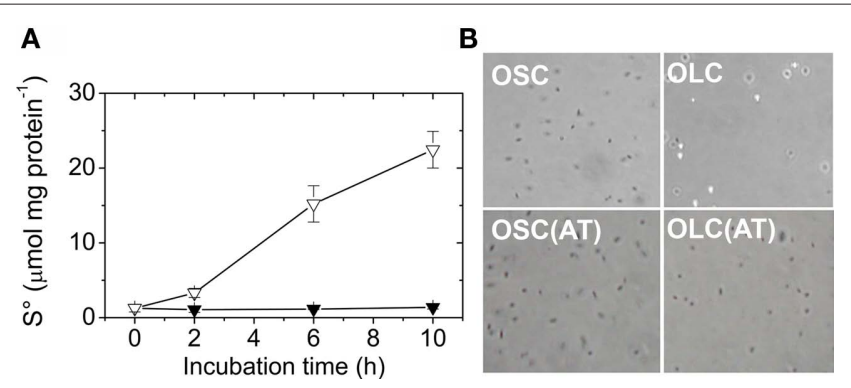

FIGURE 3 | Extracellular A. ferrooxidans deposits contain elemental sulfur. (A) Time course of elemental sulfur production by $A$. ferrooxidans cultures subjected to OSC- $(\boldsymbol{\nabla})$ or OLC- $(\nabla)$ incubations. Aliquots of $A$. ferrooxidans cultures were taken at the times indicated and cells and sulfur were sedimented by centrifugation. Sulfur was extracted from the pellets with acetone and determined by cyanolysis. (B) Phase-contrast micrographies of $A$. ferrooxidans cells (600 × magnification) after $10 \mathrm{~h}$ OLC- or OSC-incubation. Panels labeled AT correspond to cells obtained after resuspending acetonetreated pellets in DSMZ 71 medium (without thiosulfate). and Northern-Blot analysis indicated the downregulation of both doxDA1 and doxDA2 genes in OLC-incubated cells (Beard and Jerez, results not shown). doxDA1 and doxDA2 coded in $A$. ferrooxidans ATCC 23270 genome are ortholog genes of Acidianus ambivalens thiosulfate:quinone oxidoreductase (TQO; Müller et al., 2004). On the other hand, tetrathionate concentration in the culture media decreased both in OSC- and OLC-incubated cultures (Figure 5B). Surprisingly, TTH activity found in OLC-cells was c.a. 25 times lower than the one in the OSC-incubated cells $(0.007 \pm 0.004$ and $0.167 \pm 0.05 \mathrm{U} \mathrm{mg}$ protein $^{-1}$, respectively; Figure 5D). To assess whether the decrease in TTH activity was due to a possible transcriptional regulation of tet $H$ gene, its transcription was determined by qRT-PCR. Transcriptional levels of tet $H$ were highly downregulated in A. ferrooxidans cells subjected to OLC-incubations during 5-20 h (Figure 5E). A set of housekeeping genes has been proposed for qRTPCR transcriptome analysis in A. ferrooxidans (Nieto et al., 2009). However, none of those genes have been tested under the OSC/OLCconditions used in this study. Although $16 \mathrm{~S}$ rRNA transcript levels can vary due to growth conditions, we have normalized the qRTPCR values by using $16 \mathrm{~S}$ rRNA as previously reported (Vera et al., 2008; Navarro et al., 2009). The very low TTH activity and expression level of the TTH coding gene in OLC-incubated microorganisms suggest cells do not have enough TTH activity to account for either the decrease in extracellular tetrathionate concentration or the sulfur globule production observed. Since hydrolysis of tetrathionate by a TTH is proposed as the key step during biological formation of hydrophillic sulfur globules (Steudel, 2003), lack of TTH activity in OLC-incubated cells was unexpected and suggests the existence of a mechanism independent of cellular TTH activity for generation of sulfur globules by $A$. ferrooxidans.

\section{TTH ACTIVITY IS PRESENT IN A. FERROOXIDANS CULTURE SUPERNATANTS}

Due to the extracellular nature of sulfur globules produced by $A$. ferrooxidans, supernatant fractions from OSC- and OLC-incubated cultures were further investigated. To determine the low molecular weight thiol composition of supernatants from A. ferrooxidans

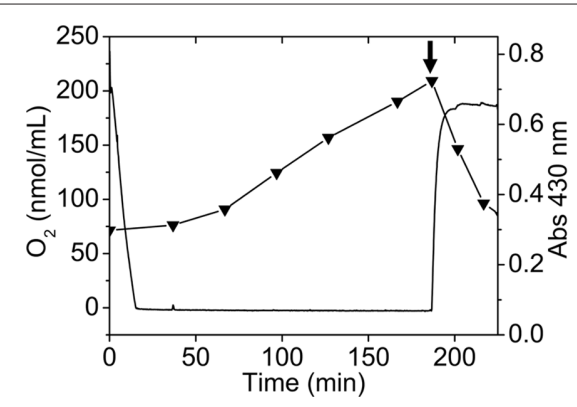

FIGURE 4 | Production of sulfur globules depend on oxygen concentration present in the culture medium. Oxygen concentration (continuous line) was determined by using a liquid-phase oxygraph and absorbance at $430 \mathrm{~nm}(\boldsymbol{\nabla})$ of aliquots taken at the indicated times was measured in a spectrophotometer. The oxygraph-cell was filled with $2.5 \mathrm{~mL}$ of a thiosulfate-grown $A$. ferrooxidans culture and OLC-incubation was started by closing the oxygraph-cell lid. OSC was obtained by removing the oxygraph-cell lid and leaving $1.0 \mathrm{~mL}$ of the $A$. ferrooxidans culture in the oxygraph-cell (shown by an arrow). Oxygraph stirrer was set at 100 RPM. 


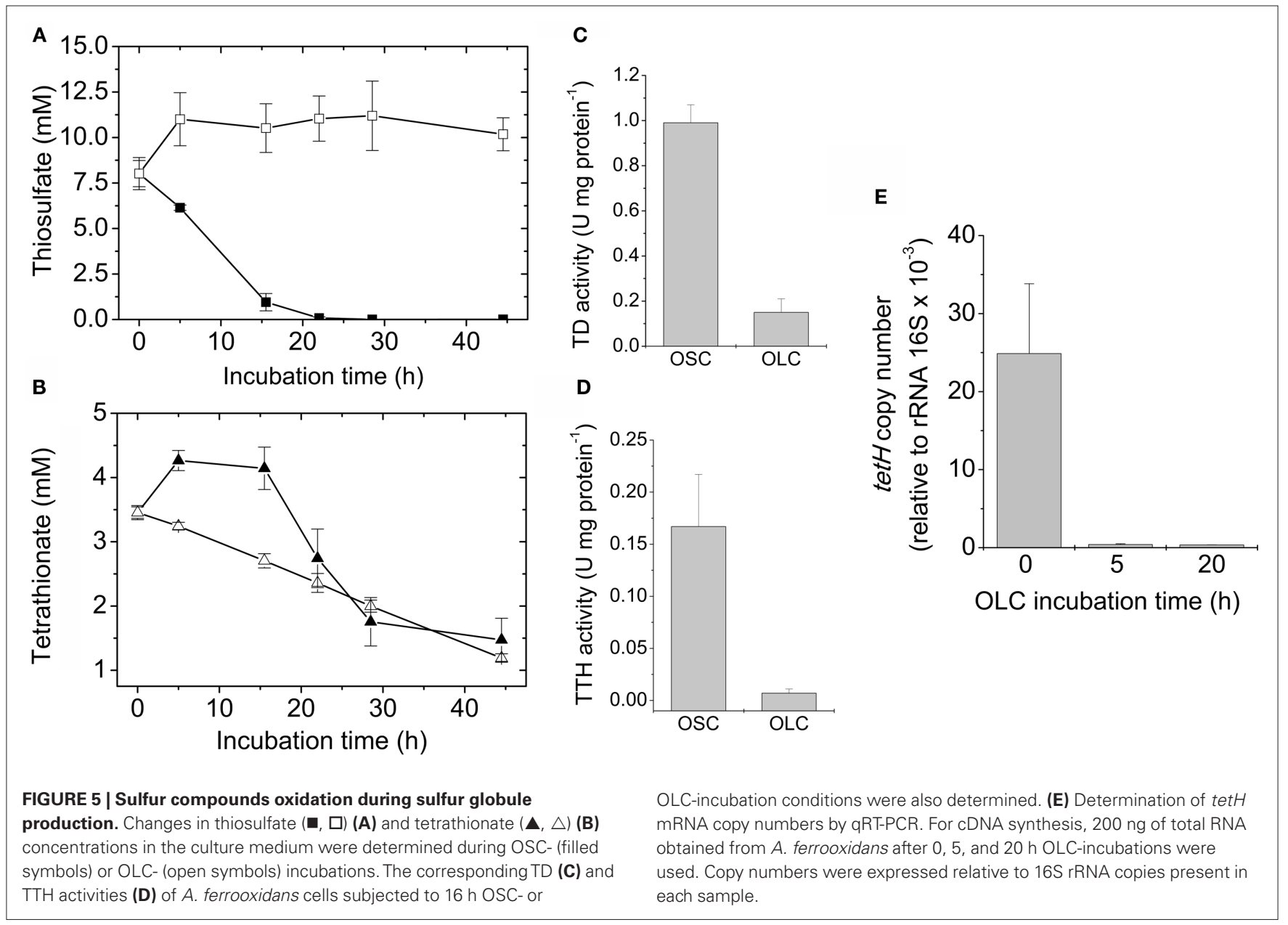

cultures subjected to OLC-incubation, they were directly derivatized with monobromobimane and analyzed by HPLC. Unlike OSC culture supernatants, the OLC-incubated $A$. ferrooxidans supernatants showed the presence of sulfite (Figure 6A). During the incubation of $A$. ferrooxidans cultures under OLC sulfite accumulated in the supernatants (Figure 6B). Due to the oxygen requirement for sulfur-oxidizing enzymes from thiobacilli (Suzuki, 1994) it is not likely that sulfite arose from elemental sulfur oxidation under OLC-incubation. TTH activity was detected both in OSC- and OLC-incubated culture supernatants (Figure 6C). The proteins present in the growth medium of OLC-incubated cells were precipitated and analyzed by SDS-PAGE (Figure 6D). Two of the three main protein bands present in the SDS-PAGE could be correctly identified by MALDI-TOF/MS (Table 1).

Protein band No. 1 corresponded to TetH protein of A. ferrooxidans ATCC 53993, which is identical to TetH from $A$. ferrooxidans ATCC 23270 used in this study and actually corresponds to A. ferrooxidans TTH (Buonfiglio et al., 1999; Kanao et al., 2007). Although TetH has a predicted Sec-type signal peptide, to date its subcellular location is not clear.

Protein band No. 2 was identified as the phosphate-binding protein PstS2 of A. ferrooxidans ATCC 23270. This protein belongs to the phosphate starvation responding Pho regulon of this microorganism (Seeger and Jerez, 1993; Vera et al., 2003). Phosphate-binding proteins have also been reported in the medium of other bacteria (Tjalsma et al., 2004; Diaz et al., 2005; Meneses et al., 2010). Besides its obvious role in phosphate uptake, its possible relationship with extracellular Tet $\mathrm{H}$ function in $A$. ferrooxidans cannot be anticipated at present.

The evidence presented here suggests that the TetH protein found in A. ferrooxidans culture supernatants corresponded to an active TTH and its activity would be related to extracellular sulfur globule production under OLC-incubations.

\section{DISCUSSION}

Thiosulfate is relatively unstable at $\mathrm{pH}$ values below 4.0. As the culture medium becomes acid due to thiosulfate oxidation, sulfite, and elemental sulfur formation is usually observed. For this reason it has been suggested that batch cultures are not adequate to study growth of thiobacilli on thiosulfate (Pronk et al., 1990). However, when washed $A$. ferrooxidans cells are used as inoculum at low cell densities $\left(2.5 \times 10^{6}\right.$ cells $\left./ \mathrm{mL}\right)$, neither the precipitation of elemental sulfur nor the formation of sulfur globules take place in batch cultures. Therefore, we have used these conditions to study the effect of oxygen availability in thiosulfate-grown A. ferrooxidans cells. 
A

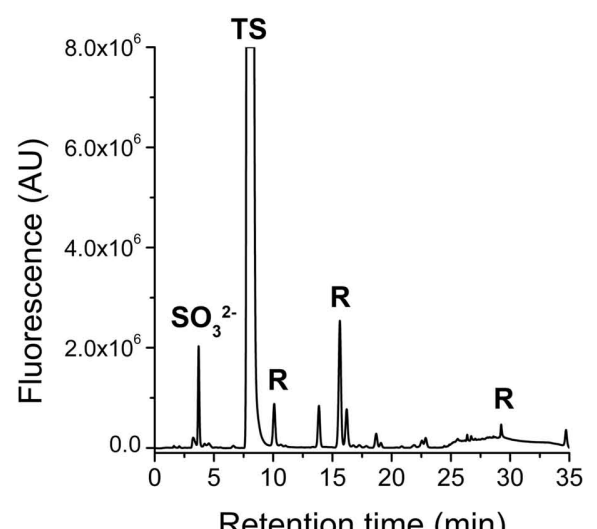

C

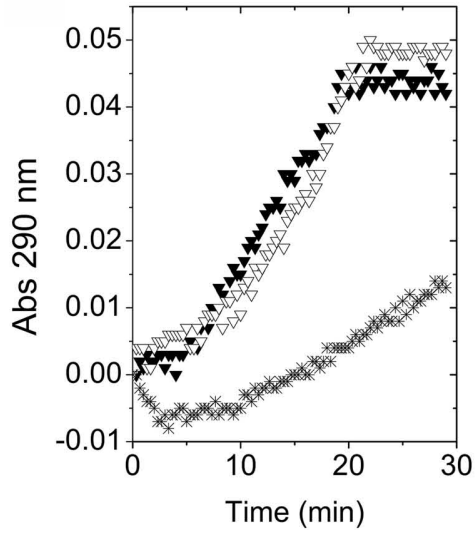

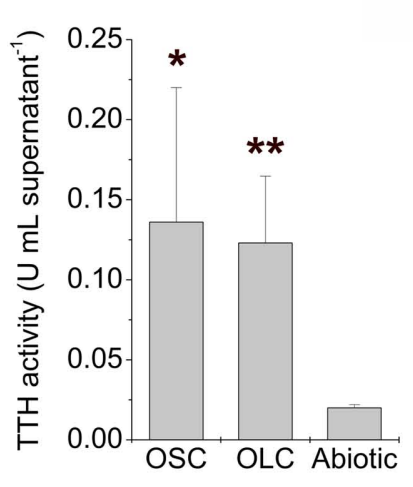

B

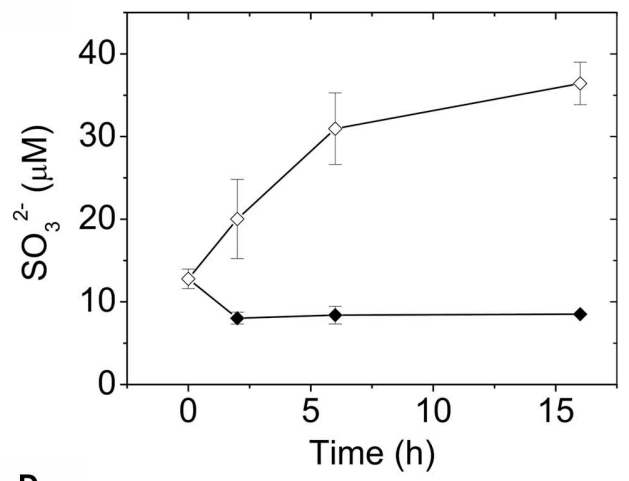

D

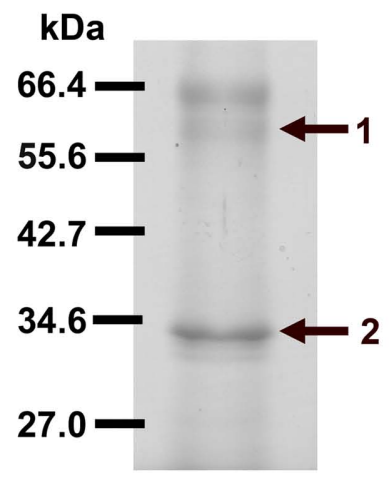

FIGURE 6 |Tetrathionate hydrolase activity is present in A. ferrooxidans culture supernatants. (A) Identification of low molecular weight thiols in A. ferrooxidans culture supernatants by HPLC. Thiols present in the culture supernatants from $A$. ferrooxidans subjected to $5 \mathrm{~h}$ OLC-incubation were derivatized with the monobromobimane reagent and separated by using HPLC. $\mathrm{SO}_{3}^{2-}$, sulfite; TS, thiosulfate; $\mathrm{R}$, reagent or water-derived peaks. (B) Changes in sulfite concentrations in thiosulfate-grown $A$. ferrooxidans culture supernatants during OSC- $(\bullet)$ or OLC- ( () incubations. Aliquots were taken from cultures at the indicated times, derivatized with monobromobimane reagent, and analyzed by HPLC. Sulfite concentrations were determined by integration of the area corresponding to the sulfite peak on each chromatogram. (C) (Left panel) Cells were separated from their growth medium by centrifugation and the filtered supernatants were used directly for TTH activity measurements.
Spectrophotometer original traces [(A), $290 \mathrm{~nm}$ ] from representative continuous TTH activity assays of supernatants from thiosulfate-grown $A$. ferrooxidans cultures subjected to $16 \mathrm{~h}$ OSC- $(\boldsymbol{\nabla})$ or OLC- $(\nabla)$ incubations. The Abiotic control (*) corresponds to the tetrathionate-dependent increase in absorbance at $290 \mathrm{~nm}$ when sterile DSMZ 71 medium (without thiosulfate) was used instead of culture supernatants in the TTH assay. (Center panel), TTH activity (expressed as $U \mathrm{~mL}$ supernatant ${ }^{-1}$ ) from $A$. ferrooxidans culture supernatants. Statistically significative differences (Student's t-test; $n=3$; *, $p$ value $<0.05 ;{ }^{* *}, p$ value $<0.005$ ) are indicated. (D) Identification of TetH protein in supernatants from $A$. ferrooxidans cultures incubated under OLC. Proteins precipitated by the PRMM method were separated by SDS-PAGE and stained with Coomassie Blue G-250. The bands obtained were isolated from the gel and identified by mass spectrometry analysis. The protein band corresponding to the tetH gene product is indicated by an arrow.

Table 1 | Proteins present in OLC-incubated $A$. ferrooxidans growth media identified by MALDI-TOF/MS.

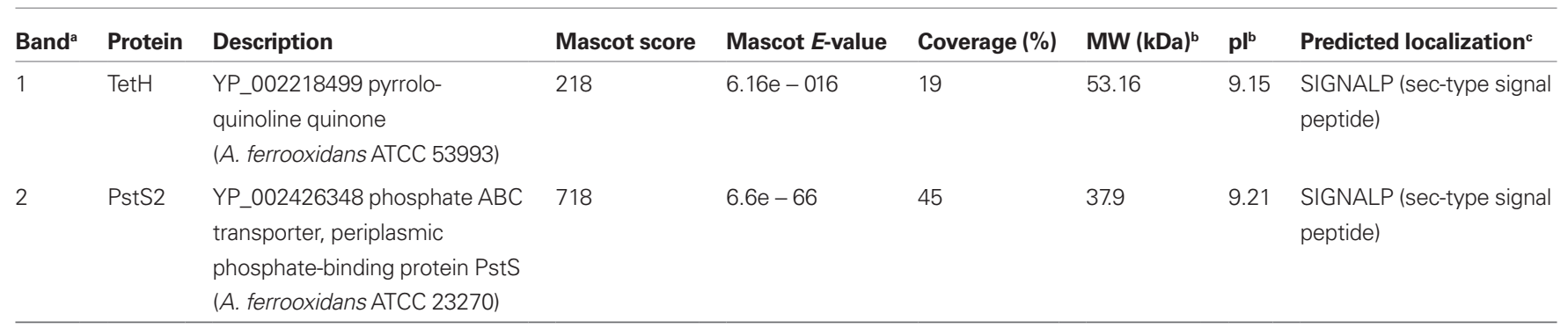

${ }^{a}$ Band numbers according to Figure $6 \boldsymbol{D}$.

${ }^{b}$ Deduced from aminoacidic sequences.

'Subcellular localization predicted by using the SubCell 1.0 server (http://www.cbs.dtu.dk/services/SubCell/). 
Cell densities did not change under OLC, indicating that cells did not grow or lyse during the conditions used in the experiments. Therefore, it is not likely that sulfur globules were released to the culture medium by a cell-lysis mechanism, as it has been proposed in Thermoanaerobacterium sulfurigignens JW/SL-NZ826 ${ }^{\mathrm{T}}$ (Lee et al., 2007). To assess whether the lack of oxygen affects in general the gene expression pattern in A. ferrooxidans during OLC-incubations, transcriptional levels of a group of genes related to stress response and other cellular processes were measured by using a DNA-microarray system as described before (Orell et al., 2010). Levels of transcripts of most A. ferrooxidans genes measured showed no significant differences. The only significant change observed in A. ferrooxidans cultures subjected to OLC-incubations was the downregulation of the $c y d A$ gene coding for a subunit of a $b d$-type terminal quinol-oxidase, suggesting that cells were actually responding rather specifically to changes in the availability of oxygen under the experimental set-up used (results not shown).

To date the subcellular location of TetH is not clear. It has been previously described as an outer membrane protein (Buonfiglio et al., 1999), a soluble and probably periplasmic protein (de Jong et al., 1997; Chi et al., 2007), and as a membrane-associated protein (Kanao et al., 2007). The gene coding for TetH contains a Sec-secretion signal (Kanao et al., 2007), strongly suggesting that it is a protein secreted into the periplasm (Table 1). The extracellular location of TetH reported here corresponds to a new finding and opens the question about the mechanism involved in its secretion from the periplasm into the growth medium. The sequence of the $\mathrm{N}$-terminal end of the protein was not obtained during its identification by mass spectrometry in our studies. Therefore the cleavage site of the Sec-type signal peptide could not be confirmed in the extracellular TetH. Nevertheless, bioinformatic analyses have shown that $A$. ferrooxidans ATCC 23270 genome contains genes coding for six of the eight secretion systems known in bacteria (Pagliai and Jerez, 2009), several of which would be capable of secreting proteins by using the Sec system.

The current model for thiosulfate oxidation by the S4I-pathway (Pronk et al., 1990; Ghosh and Dam, 2009) explains intracellular sulfur globule production as some of the disulfane monosulfonic acid derived from periplasmic tetrathionate oxidation can follow the "higher polythionates pathway" that leads to the formation of intracellular sulfur deposits (Hazeu et al., 1988; Figure 7). However, this model does not explain the deposition of extracellular sulfur by A. ferrooxidans shown in this report. Since TTH activity was found in the extracellular medium of both OSC- and OLC-conditions, sulfur deposition was expected in both kinds of cultures. However, sulfur globule formation was seen only under OLC-conditions. The extracellular TTH activity could account for tetrathionate disappearance from the growth medium. The concomitant extracellular sulfur globule production in OLC-incubated A. ferrooxidans cells could be explained by the negligible periplasmic TD and TTH activities. Under these conditions, the only reaction that could take place is the extracellular hydrolysis of tetrathionate (Figure 7). An additional possible explanation for the lack of sulfur globules in OSC-incubated cells is that sulfur is indeed produced by extracellular TTH but is followed by its immediate oxidation by the cells.

To understand the significance of sulfur globule generation under OLC-incubations, some issues regarding enzymatic activities as well as some physiological considerations must be taken into account. The lack of TD activity in OLC-incubated cells is probably due to the fact that in A. ferrooxidans this activity should be directly coupled to molecular oxygen reduction by a terminal oxidase, as it has been shown in A. ambivalens (Müller et al., 2004). A. ferrooxidans is a facultative anaerobe bacterium and can use ferric iron as an electron acceptor during anaerobic oxidation of elemental sulfur (Pronk et al., 1992). Moreover, it was recently reported that A. ferrooxidans TTH has ferric iron reductase activity (Sugio et al., 2009). Although ferric iron may act as an alternative electron acceptor in anoxygenic tetrathionate oxidation, the physiological electron acceptors for this reaction are unknown. Once $A$. ferrooxidans cells switch to anaerobic metabolism, elemental sulfur stored into sulfur globules may be further oxidized by means of ferric iron respiration.

Our preliminary observations indicate that TetH protein is also present in supernatants obtained from elemental sulfur and pyritegrown A. ferrooxidans cultures, suggesting that extracellular TTH activity may play a role during elemental sulfur oxidation and bioleaching of sulfide ores. In this regard, it is noteworthy that during pyrite oxidation in acidic environments and in excess ferric iron, thiosulfate is formed as the key intermediary (Schippers et al., 1996). The rate of chemical oxidation of thiosulfate is several orders of magnitude faster than that for tetrathionate oxidation. Therefore, tetrathionate can persist in the environment and may be metabolized by sulfur-oxidizing microorganisms (Druschel et al., 2003). This gives a geochemical relevance to the biochemical pathways related to tetrathionate oxidation. Interestingly, the production of colloidal sulfur particles within A. ferrooxidans capsule was observed during incubation of cells grown on synthetic pyrite films (Rojas-Chapana et al., 1996).

To the best of our knowledge, the present report is the first evidence for an enzymatic activity existing in $A$. ferrooxidans culture supernatants. Furthermore, this finding suggests a mechanism for extracellular sulfur globule deposition in a microorganism that does not use the branched-PSO pathway for thiosulfate oxidation. In addition, a physiological role for $A$. ferrooxidans extracellular sulfur globule production is proposed as a response allowing cells to overcome a

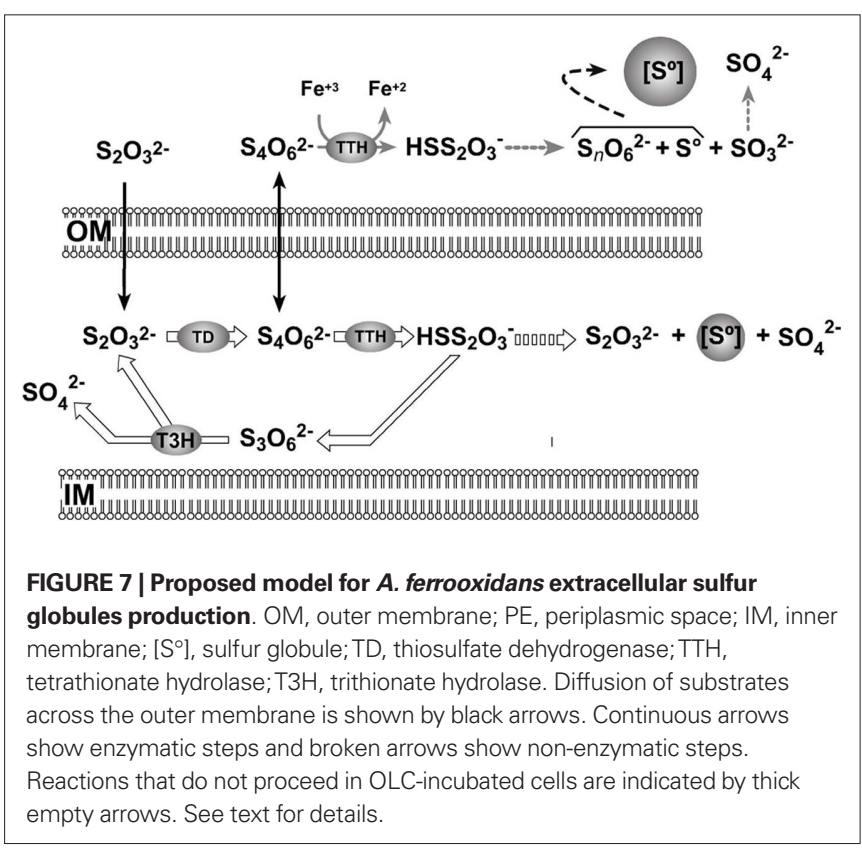


momentary decrease in oxygen availability, probably as an early step during the metabolic switch to anaerobic lifestyle. Further investigation using non-invasive methods for in situ analysis of sulfur globules (such as sulfur K-edge XANES or Laser Raman spectroscopy) will be needed in order to unveil the exact chemical nature of the sulfur species generated by A. ferrooxidans in its extracellular sulfur globules and whether they are different from the composition previously reported for A. ferrooxidans globules (Prange et al., 2002). In this regard, a recent study on sulfur speciation using sulfur K-edge XANES shows that the extracellular globules from thiosulfate-grown A. ferrooxidans are composed mainly of elemental sulfur rings ( $\mathrm{He}$ et al., 2010), which is consistent with the results reported here.

\section{CONCLUSION}

Acidithiobacillus ferrooxidans produce extracellular sulfur globules when thiosulfate-grown cultures are shifted to OLC. The finding of TetH protein in the $A$. ferrooxidans growth medium represent a

\section{REFERENCES}

Buonfiglio, V., Polidoro, M., Soyer, F., Valenti, P., and Shively, J. (1999). A novel gene encoding a sulfur-regulated outer membrane protein in Thiobacillus ferrooxidans. J. Biotechnol. 72, 85-93.

Caldwell, R. B., and Lattemann, C. T. (2004). Simple and reliable method to precipitate proteins from bacterial culture supernatant. Appl. Environ. Microbiol. 70, 610-612.

Chi, A., Valenzuela, L., Beard, S., Mackey, A. J., Shabanowitz, J., Hunt, D. F., and Jerez, C. A. (2007). Periplasmic proteins of the extremophile Acidithiobacillus ferrooxidans: a high throughput proteomics analysis. Mol. Cell. Proteomics 6, 2239-2251.

de Jong, G. A. H., Hazeu, W., Bos, P., and Kuenen, J.G. (1997). Polythionate degradation by tetrathionate hydrolase of Thiobacillus ferrooxidans. Microbiology 143, 499-504.

Diaz, M., Esteban, A., Fernandez-Abalos, J.M., and Santamaria, R. I. (2005). The high-affinity phosphate-binding protein PstS is accumulated under high fructose concentrations and mutation of the corresponding gene affects differentiation in Streptomyces lividans. Microbiology 151, 2583-2592.

Druschel, G. K., Hamers, R. J., and Banfield, J. F. (2003). Kinetics and mechanism of polythionate oxidation to sulfate at low $\mathrm{pH}$ by $\mathrm{O} 2$ and $\mathrm{Fe}^{3+}$. Geochim. Cosmochim. Acta 67, 4457-4469.

Friedrich, C. G., Bardischewsky, F., Rother, D., Quentmeier, A., and Fischer, J. (2005). Prokaryotic sulfur oxidation. Curr. Opin. Microbiol. 8, 253-259.

George, G. N., Gnida, M., Bazylinski, D. A., Prince, R. C., and Pickering, I. J. (2008). X-ray absorption spectroscopy as a probe of microbial sulfur

novel issue regarding both the subcellular location and the function of this enzyme, suggesting its involvement in an extracellular sulfur globule deposition mechanism in a microorganism that does not use the branched-PSO pathway for RISCs oxidation. Although demonstration of the mechanism proposed here will require further studies with purified TTH, we believe it may have a physiological as well as geochemical relevance during sulfur compounds cycling in microaerophilic metal sulfide rich environments, in which $A$. ferrooxidans can be normally found.

\section{ACKNOWLEDGMENTS}

This work was supported by ICM-P05-001-F project and a doctoral fellowship from CONICYT to Simón Beard. We thank A. Hokeer for her help with the English language and S. Rubio and F. Perez (Laboratory of Vegetal Biochemistry, Faculty of Sciences, University of Chile) for their support in the oxygraph measurements and A. Banderas for encouraging us to start this work.

and tetrathionate. Anal. Chem. 41 898-902.

Kelly, D. P., Shergill, J. K., Lu, W. P., and Wood, A.P.(1997). Oxidative metabolism of inorganic sulfur compounds by bacteria. Antonie Van Leeuwenhoek 71, 95-107.

Kelly, D. P., and Wood, A. P. (1998). "Microbes of the sulfur cycle," in Techniques in Microbial Ecology, eds R. S. Burlage, R. Atlas, D. Stahl, G Geesey, and G. Sayler (New York: Oxford University Press), 31-57.

Kletzin, A. (1989). Coupled enzymatic production of sulfite, thiosulfate, and hydrogen sulfide from sulfur: purification and properties of a sulfur oxygenase reductase from the facultatively anaerobic archaebacterium Desulfurolobus ambivalens. J. Bacteriol. 171, 1638-1643.

Lee, Y. J., Prange, A., Lichtenberg, H., Rohde, M., Dashti, M., and Wiegel, J. (2007). In situ analysis of sulfur species in sulfur globules produced from thiosulfate by Thermoanaerobacter sulfurigignens and Thermoanaerobacterium thermosulfurigenes. J. Bacteriol. 189, 7525-7529.

Meneses, N., Mendoza-Hernandez, G., and Encarnacion, S. (2010). The extracellular proteome of Rhizobium etli $\mathrm{CE} 3$ in exponential and stationary growth phase. Proteome Sci. 8, 51 .

Müller, F. H., Bandeiras, T. M., Urich, T., Teixeira, M., Gomes, C. M., and Kletzin, A. (2004). Coupling of the pathway of sulphur oxidation to dioxygen reduction: characterization of a novel membrane-bound thiosulphate:quinone oxidoreductase. Mol. Microbiol. 53, 1147-1160.

Navarro, C. A., Orellana, L. H., Mauriaca, C., and Jerez, C. A. (2009). Transcriptional and functional studies of Acidithiobacillus ferrooxidans genes related to survival in the presence of copper. Appl. Environ. Microbiol. 75, 6102-6109.

Nieto, P.A., Covarrubias, P.C., Jedlicki, E., Holmes, D. S., and Quatrini, R. (2009). Selection and evaluation of reference genes for improved interrogation of microbial transcriptomes: case study with the extremophile Acidithiobacillus ferrooxidans. BMC Mol. Biol. 10, 63. doi: 10.1186/1471-2199-10-63

Olson, G. J., Brierley, J. A., and Brierley, C. L. (2003). Bioleaching review part B: progress in bioleaching: applications of microbial processes by the minerals industries.Appl. Microbiol.Biotechnol. 63, 249-257.

Orell, A., Navarro, C. A., Arancibia, R., Mobarec, J. C., and Jerez, C. A. (2010). Life in blue: copper resistance mechanisms of bacteria and archaea used in industrial biomining of minerals. Biotechnol. Adv. 28, 839-848.

Pagliai, F. A., and Jerez, C. A. (2009). The secretome of the extremophilic Acidithiobacillus ferrooxidans ATCC 23270. Adv. Mat. Res. 71-73, 183-186.

Prange, A., Chauvistre, R., Modrow, H., Hormes, J., Truper, H. G., and Dahl, C. (2002). Quantitative speciation of sulfur in bacterial sulfur globules: $\mathrm{X}$-ray absorption spectroscopy reveals at least three different species of sulfur. Microbiology 148, 267-276.

Pronk, J. T., de Bruyn, J. C., Bos, P., and Kuenen, J. G. (1992). Anaerobic growth of Thiobacillus ferrooxidans. Appl. Environ. Microbiol. 58, 2227-2230.

Pronk, J. T., Meulenberg, R., Hazeu, Z., Bos, P., and Kuenen, J. G. (1990). Oxidation of inorganic sulphur compounds by acidophilic thiobacilli. FEMS Microbiol. Rev. 75, 293-306.

Rawlings, D. E. (2005). Characteristics and adaptability of iron- and 
sulfur-oxidizing microorganisms used for the recovery of metals from minerals and their concentrates. Microb. Cell Fact. 4, 13.

Rethmeier, J., Rabenstein, A., Langer, M., and Fischer, U. (1997). Detection of traces of oxidized and reduced sulfur compounds in small samples by combination of different highperformance liquid chromatography methods. J. Chromatogr. A 760, 295-302.

Rohwerder, T., and Sand, W. (2003). The sulfane sulfur of persulfides is the actual substrate of the sulfur-oxidizing enzymes from Acidithiobacillus and Acidiphilium spp. Microbiology 149, 1699-1710.

Rojas-Chapana, J. A., Giersig, M., and Tributsch, H. (1996). The path of sulfur during the bio-oxidation of pyrite by Thiobacillus ferrooxidans. Fuel 75, 923-930.

Schippers, A., Jozsa, P., and Sand, W. (1996). Sulfur chemistry in bacterial leaching of pyrite. Appl. Environ. Microbiol. 62, 3424-3431.
Seeger, M., and Jerez, C. A. (1993). Phosphate-starvation induced changes in Thiobacillus ferrooxidans. FEMS Microbiol. Lett. 108, 35-41.

Steudel, R. (2003). Aqueus sulfur sols. Top. Curr. Chem. 230, 153-166.

Steudel, R., Holdt, G., Göbel, T., and Hazeu, W. (1987). Chromatographic separation of higher polythionates SnO62- $(n=3 \ldots 22)$ and their detection in cultures of Thiobacillus ferrooxidans: molecular composition of bacterial sulfur secretion. Angew. Chem. Int. Ed. Engl. 26, 151-153.

Sugio, T., Taha, T. M., and Takeuchi, F. (2009). Ferrous iron production mediated by tetrathionate hydrolase in tetrathionate-, sulfur-, and irongrown Acidithiobacillus ferrooxidans ATCC 23270 cells. Biosci. Biotechnol. Biochem. 73, 1381-1386.

Suzuki, I. (1994). Sulfur-oxydazing enzymes. Meth. Enzymol. 243, 455-462.

Tjalsma, H., Antelmann, H., Jongbloed, J. D., Braun, P. G., Darmon, E., Dorenbos, R., Dubois, J. Y., Westers,
H., Zanen, G., Quax, W. J., Kuipers, O. P., Bron, S., Hecker, M., and van Dijl, J. M.. (2004). Proteomics of protein secretion by Bacillus subtilis: separating the "secrets" of the secretome. Microbiol. Mol. Biol. Rev. 68, 207-233.

Varela, C., Mauriaca, C., Paradela, A., Albar, J. P., Jerez, C. A., and Chávez, F. P. (2010). New structural and functional defects in polyphosphate deficient bacteria: a cellular and proteomic study. BMC Microbiol. 10, 7. doi: 10.1186/1471-2180-10-7

Vera, M., Guiliani, N., and Jerez, C. A. (2003). Proteomic and genomic analysis of the phosphate starvation response of Acidithiobacillus ferrooxidans. Hydrometallurgy 71, 125-132.

Vera, M., Pagliai, F., Guiliani, N., and Jerez, C.A. (2008). The chemolithoautotroph Acidithiobacillus ferrooxidans can survive under phosphate-limiting conditions by expressing a C-P lyase operon that allows it to grow on phosphonates. Appl.Environ. Microbiol.74, 1829-1835.
Conflict of Interest Statement: The authors declare that the research was conducted in the absence of any commercial or financial relationships that could be construed as a potential conflict of interest.

Received: 05 January 2011; accepted: 04 April 2011; published online: 18 April 2011. Citation: Beard S, Paradela A, Albar JP and Jerez CA (2011) Growth of Acidithiobacillus ferrooxidans ATCC 23270 in thiosulfate under oxygen-limiting conditions generates extracellular sulfur globules by means of a secreted tetrathionate hydrolase. Front. Microbio. 2:79. doi: 10.3389/ fmicb.2011.00079

This article was submitted to Frontiers in Microbial Physiology and Metabolism, a specialty of Frontiers in Microbiology. Copyright (C) 2011 Beard, Paradela, Albar and Jerez. This is an open-access article subject to a non-exclusive license between the authors and Frontiers Media SA, which permits use, distribution and reproduction in other forums, provided the original authors and source are credited and other Frontiers conditions are complied with. 